\title{
La confiance vigilante : les instruments de la saine gouvernance
}

\author{
Jeanne Simard, LL.D., F. Adm.A., professeure titulaire \\ Marc-André Morency, sociologue, professeur retraité \\ Laboratoire de recherche et d'intervention en gouvernance des organisations (LARIGO) \\ Université du Québec à Chicoutimi
}

\begin{abstract}
"Faire confiance à un escroc, ce n'est pas une vertu, c'est une faute. Faire confiance à un inconnu, ce n'est pas une vertu, c'est une imprudence. ${ }^{I}$."
\end{abstract}

\section{INTRODUCTION}

Les travaux de la Commission d'enquête sur l'octroi et la gestion des contrats publics dans l'industrie de la construction (Commission Charbonneau) mettent en lumière que de nombreux professionnels, de nombreux gestionnaires, n'ont pas été à la hauteur de leurs responsabilités fiduciaires, de leurs contrats moraux, non seulement envers leurs clientèles, mais aussi envers les collectivités locales et nationales.

Les travaux de la Commission d'enquête sur l'octroi et la gestion des contrats publics dans l'industrie de la construction (Commission Charbonneau) mettent en lumière que de nombreux professionnels, de nombreux gestionnaires, n'ont pas été à la hauteur de leurs responsabilités fiduciaires, de leurs contrats moraux, non seulement envers leurs clientèles, mais aussi envers les collectivités locales et nationales. La population québécoise en est révoltée, choquée. Peuton lui donner tort? La crise en cours depuis 2007 est un symptôme encore plus évident que le monde politique s'est montré jusqu'ici incapable de réguler la sphère financière, de restaurer une saine économie. Les économistes et les analystes les plus qualifiés ont de fait montré que nos États-gardiens auraient besoin d'un gardien (Quis custodiet ipsos custodes? disait le caustique auteur latin Juvénal ${ }^{2}$ ); ils décrivent comment on a toléré sinon encouragé les dérives d'une finance planétaire au caractère mafieux, comment on a laissé s'accroitre les inégalités sociales et très peu fait en somme pour restaurer la foi dans les institutions ${ }^{3}$. Le lien de confiance entre les dirigeants, souvent des professionnels, et la population, souffre d'un déficit qui inquiète à juste titre de nombreux observateurs. Mais cette crise annonce la fin, pour plusieurs, d'une certaine forme de confiance confinant à l'insouciance, ce qui n'est pas une mauvaise chose en soi; on assisterait à l'éveil d'une confiance plus vigilante et d'un esprit qui se veut plus critique.

Dans cet article, nous allons, dans un premier temps, faire ressortir le caractère crucial de la confiance, vue comme prudence réfléchie, en tant qu'opérateur de la socialité. Le résultat de deux sondages, dont l'un commandé en 2012 par le Conseil interprofessionnel du Québec (CIQ), sur la confiance qu'entretiennent les Québécois envers les professionnels, et l'autre, récurrent, portant sur les valeurs des Québécois, nous montrent des signes évidents de désaffection de la population vis-à-vis des dirigeants institutionnels. Dans un deuxième temps, nous examinerons les processus en cause et les principes au fondement de la responsabilité sociale ou collective des gestionnaires qui permettraient de restaurer la confiance, idéalement sous forme de prudence réfléchie. Nous verrons sur quelles bases ces principes et processus pourraient contribuer activement aux besoins de développement socioéconomique d'une population qui se montre de plus en plus fataliste, qui a le sentiment d'une perte de contrôle, des forces impersonnelles s'étant emparées de la trame de l'histoire et de leur vie. Une éthique de la bienveillance (du «care») sera examinée comme une avenue intéressante dans le renouveau de la gouvernance et de la participation. 
Enfin, nous verrons que le développement de la confiance exigera une modification de la formation initiale et continue des gestionnaires et ce, dans la perspective de la mise en place de pratiques de saine gouvernance. Pour les aider dans leur travail, les gestionnaires devront faire davantage appel à des outils d'intervention développés par les sciences sociales, afin de réduire le caractère faussement impersonnel de la prise de décision et de la gouvernance.

\section{POLYMATHIE DE LA NOTION DE CONFIANCE}

Toute chose ayant son bon côté, la crise en cours a pour mérite de faire ressortir le caractère crucial de la confiance comme opérateur central de la socialité. Le latin nous a permis de donner un nom à cette dimension cruciale: con-fidere : cum, « avec » et fidere « fier ». Cela signifie simplement qu'on confie quelque chose de précieux à quelqu'un, en se fiant à lui, en s'abandonnant ainsi à sa bienveillance et à sa bonne foi ${ }^{4}$. La confiance aveugle peut alors se confondre avec l'insouciance. Elle peut dans ce cas de figure devenir, comme le mentionne André Comte-Sponville, une faute, si on se trouve à faire confiance à un escroc, ou une imprudence, si l'on fait confiance à un inconnu. Cet «élan vers l'indifférence », ce déficit de la raison pratique, peut avoir de graves consé- quences sur la destinée individuelle ou collective. À l'autre extrémité du spectre, une méfiance aveugle et systématique envers toutes les institutions, dans la vie courante, serait tout aussi néfaste, s'apparentant cette fois à la paranoïa. Cependant, le monde est beaucoup trop complexe et possède trop de virtualités pour qu'un individu soit en mesure de comprendre et d'évaluer toute la portée de ses actes. Selon Niklas Luhmann, «[...] s'il ne faisait pas confiance de manière courante, il n'arriverait même pas à quitter son lit le matin. Une angoisse indéterminée, une répulsion paralysante, l'assailliraient ${ }^{5}$. Il convient de réaliser que la notion de confiance et ses variantes occupent un continuum, que représente la figure 1.

\section{Figure 1 - Continuum des niveaux de confiance}

\begin{tabular}{|c|c|c|c|c|}
\hline $\begin{array}{l}\text { Confiance } \\
\text { aveugle } \\
\text { (insouciance) }\end{array}$ & $\begin{array}{l}\text { confiance } \\
\text { imprudente }\end{array}$ & $\begin{array}{l}\text { confiance } \\
\text { vigilante } \\
\text { ou } \\
\text { prudence réfléchie }\end{array}$ & méfiance & $\begin{array}{l}\text { méfiance } \\
\text { systématique } \\
\text { (paranoïa) }\end{array}$ \\
\hline
\end{tabular}

$\mathrm{Au}$ plan théorique, en fonction du cadre de référence ou du paradigme employé, les diverses disciplines tendent à apporter leur interprétation particulière de cette notion, d'où la polymathie que l'on rencontre dans la littérature ${ }^{6}$. En psychologie, en biologie, la confiance évoque la présence d'une émotion, d'un sentiment de sécurité, chez celui qui se fie à quelqu'un ou à quelque chose.

Certains chercheurs sont allés jusqu'à avancer qu'une hormone naturelle produite par l'hypothalamus, l'ocytocine, serait un facteur causal, un déterminant, dans la relation de confiance ${ }^{7}$. Pour l'éthicien, la confiance prend l'aspect d'une valeur mesurant la qualité de certains rapports ${ }^{8}$. Pour le sociologue et l'économiste, la confiance fait référence à un ensemble de représentations de la réalité, un état des rapports sociaux qui s'accompagne d'affects psychiques ou émotionnels. Pour eux, les institutions sont fondées sur le principe de la confiance ${ }^{9}$. C'est notamment le cas pour la valeur de la monnaie, des contrats qui nous relient, des relations entre nations, etc. ${ }^{10}$. Lorsque la confiance vient à se briser entre les êtres sociaux que nous sommes, on assiste à une crise de la socialité, à un amoindrissement de la qualité de nos rapports sociaux. Le prix Nobel d'économie 1972, Kenneth Arrow, n'a pas manqué, dans sa critique de l'économisme traditionnel, de reconnaître dans la confiance un élément central de l'activité économique et un facteur des écarts entre pays : 
«Virtuellement tout échange commercial contient une part de confiance, comme toute transaction qui s'inscrit dans la durée. On peut vraisemblablement soutenir qu'une grande part du retard de développement économique d'une société est due à l'absence de confiance réciproque entre ses citoyens ${ }^{11}$. »

Comment cette problématique affecte-t-elle notre réalité immédiate? Un sondage dévoilé à la Semaine des professionnels, qui avait lieu du 7 au 11 octobre 2012, permet d'apprécier la situation qui touche de plus près les gestionnaires québécois. Le Conseil interprofessionnel du Québec s'intéresse en effet non seulement à la situation des professions, mais aussi à leur apport dans l'évolution de la société, dans les conditions de notre socialité. À sa demande, en septembre 2012, la firme CROP réalisait un sondage sur la confiance que les Québécois entretiennent envers les ordres professionnels et ses 345000 membres $^{12}$. Ce sondage nous apprend que le taux de confiance du public envers les professionnels est plutôt élevé : $8 \%$ des répondants leur font tout à fait confiance et $69 \%$ leur font assez confiance. Mais cette confiance sera ébranlée, c'est le cas de $86 \%$ des répondants, à l'endroit d'un professionnel déjà sanctionné pour une faute. Le sondage nous apprend enfin que la confiance des Québécois à l'égard des ordres professionnels n'a pas augmenté au cours des 10 ou 20 dernières années et même que, pour $31 \%$ de la population, elle a plutôt diminué.

En février 2012, les conclusions de la
douzième édition du baromètre de la
confiance démontraient une chute
dramatique du niveau de confiance envers les
gouvernements. En effet, près de la moitié
des Canadiens (46 \%) affirment ne pas du
tout avoir confiance en ce que racontent les
leaders gouvernementaux. La cote des chefs
de direction accuse une forte baisse,
puisqu'ils figurent au dernier rang des porte-
paroles crédibles.

Un autre sondage CROP, mais portant cette fois sur les représentations des Québécois en 2012, nous apparaît très inquiétant ${ }^{13}$. Pas moins de $72 \%$ des répondants, représentatifs de la population, ne croient plus les leaders politiques, économiques ou d'affaires. C'est le fatalisme et le sentiment de perte de contrôle sur leur vie qui les envahit. En outre, $56 \%$ croient que la société est animée par des forces qui dépassent la volonté des individus. Sur le plan international, le sondage de la firme de relations publiques Edelman rend le même son de cloche. Depuis maintenant 12 ans, cette firme réalise annuellement un «baromètre de confiance » visant quatre catégories d'acteurs institutionnels au sein de la population de 25 pays, dont le Canada: les gouvernements, les entreprises, les médias et les $\mathrm{ONG}^{14}$. En février 2012, les conclusions de la douzième édition du baromètre de la confiance démontraient une chute dramatique du niveau de confiance envers les gouvernements. En effet, près de la moitié des Canadiens (46\%) affirment ne pas du tout avoir confiance en ce que racontent les leaders gouvernementaux. La cote des chefs de direction accuse une forte baisse, puisqu'ils figurent au dernier rang des porteparoles crédibles. Ainsi, les actions des entreprises seront communiquées plus efficacement par des experts ou des académiciens, par «une personne comme moi » ou des employés, lesquels figurent aux premiers rangs des porte-paroles jugés crédibles. Au niveau des autres pays sondés, il y a une croissance spectaculaire de la défiance vis-àvis des institutions. «Sur une échelle où l'indice 50 constitue la moyenne, 12 pays sont passés en dessous de la barre fatidique en 2012 au lieu de 6 en 2011. ${ }^{15}$

De telles perceptions de perte de contrôle, de méfiance (même si, à l'occasion, elle peut être fausse ou exagérée) vis-à-vis les institutions sont dangereuses, car elles entrainent diverses conséquences plus ou moins prévisibles ${ }^{16}$. William I. Thomas a formulé un véritable théorème sociologique en énonçant que «si les humains définissent des situations comme réelles, elles sont réelles de par leurs conséquences ${ }^{17}$. Un système de pensée, une prophétie autoréalisatrice modifient les comportements de telle sorte qu'ils font advenir ce qu'ils annoncent ou d'autres conséquences, du fait de l'autorité de celui qui énonce la prophétie ou par la focalisation autonome des esprits sur le « réel » ainsi affirmé. Comme on l'a constaté dans les crises de 1929 et de 2008, la rumeur de faillite d'une banque (la banque va manquer d'argent, il 
faut retirer tout de suite notre dépôt) pouvait avoir pour effet d'entraîner une ruée sur les guichets, précipitant ainsi le défaut de paiement ${ }^{18}$. Le théorème, de par sa nature même, ne s'applique pas qu'aux banques; de nombreux politiques, professionnels, gestionnaires en font actuellement le triste constat. Ils devront contribuer à redresser la situation. Toutefois comme Louis Quéré, directeur de recherche au CNRS, le rappelle, la confiance se construit dans la durée et dans la complexité; il ne convient pas de se fier plus que de raison aux modèles de calcul rationnel dans lesquels le temps est pratiquement évacué, une simplification nettement abusive des problématiques de gouvernance et de gestion. Il propose d'ailleurs que soit entrepris un nouveau volet d'investigations empiriques pour relancer la recherche sur le sujet de la confiance ${ }^{19}$. Le grand nombre de publications sur le sujet ne doit pas nous abuser: les études sur la durée en tant que déterminant de la confiance ne font que débuter.

\section{LA RESPONSABILITÉ SOCIALE DES GESTIONNAIRES}

\section{La responsabilité des professionnels et tout particulièrement des gestionnaires, dans le développement social, dans l'amélioration de la confiance de la population vis-à-vis des institutions, est extrêmement importante et ne peut être traitée de façon négligente.}

La responsabilité des professionnels et tout particulièrement des gestionnaires, dans le développement social, dans l'amélioration de la confiance de la population vis-à-vis des institutions, est extrêmement importante et ne peut être traitée de façon négligente. De fait, le gestionnaire qui assume ses responsabilités sociales joue un rôle très important qui tend à être mieux défini et apprécié, comme en font foi de nombreux codes régissant la vie professionnelle et certains codes de conduite d'entreprises ou d'organisations publiques ${ }^{20}$. Il importe de souligner que cette reconnaissance de leur rôle va bien au-delà des simples rapports entre le gestionnaire et son « client ». Ces codes font état d'attentes à l'endroit du gestionnaire, exigeant qu'il sache apprécier ses actes professionnels ou de citoyen dans la perspective d'un développement $^{21}$, d'une vie sociale où la confiance et l'intelligence collective peuvent s'accroitre. Il doit produire des définitions de situation permettant aux acteurs sociaux de quitter le domaine de la déprime et des fausses représentations; il doit inspirer des conduites, une vie sociale plus heureuse pour les compagnons de vie que nous sommes (socius, compagnon, allié, selon l'étymologie). Enfin, on attend qu'il sache apprécier les conséquences de ses actes sur la vie sociale, qu'il puisse les concevoir en interdisci- plinarité et mobiliser les acteurs, les milieux les plus divers. Bref, on espère de ces professionnels qu'ils aient la lucidité et le courage de nous amener à développer nos «capabilités » pour reprendre les termes de l'économiste et éthicien Amartya Sen ${ }^{22}$.

Plusieurs études en sciences humaines, en économie, confirment que le niveau de mal-être, la perte de confiance sont attribuables, en grande partie, à des situations dans lesquelles l'individu a l'impression de vivre une absence de réciprocité dans les échanges (trop forte inégalité) ou une absence d'autonomie et de compréhension dans la prise de décision ${ }^{23}$. Dans cette perspective, la responsabilité sociale et les interventions des gestionnaires viseront à améliorer la capacité d'autonomie, de compréhension de la réalité, des citoyens, faisant en sorte qu'ils soient mieux en mesure de mener leur vie; le but consistant à les responsabiliser, à leur donner les outils critiques plutôt que devenir les dépendants d'une « expertise » quelconque, au sein d'approches infantilisantes. La figure du «grand leader omnipotent $^{24} \gg$ n'aurait plus sa place dans la construction d'une intelligence devant être considérée comme un attribut d'une collectivité possédant la maîtrise de sa destinée. Toujours selon André Comte-Sponville :

[...] l'on ne peut lutter contre la méfiance généralisée qu'en redonnant à chacun le sens de ses responsabilités, ce qui suppose d'abord qu'il retrouve confiance en luimême : en son pouvoir de juger, de vouloir et d'agir' 25 . 
Il existe au sein de l'administration publique de nombreux mécanismes favorisant l'exercice d'un contrôle $^{26}$. Certains mécanismes ne permettent d'agir qu'a posteriori, par exemple de faire une gestion de crise en cas de mauvaise administration, mais lorsque le mal est déjà fait, dirons-nous. On pense aux mécanismes traditionnels de contrôle du Vérificateur général, les commissions d'enquête (comme les Commissions Charbonneau, Gomery, Bastarache) ou encore aux recours accordés aux citoyens, aux lois d'accès à l'information. Ces outils ont le mérite malgré tout d'ébranler la population porteuse d'une confiance aveugle ou imprudente, de favoriser un sens plus critique des situations. Les législateurs fédéral et québécois ont, par ailleurs, développé ou utilisé (encore trop peu, diront certains) des mécanismes qui agissent, cette fois, en aval des prises de décision, font la promotion d'une saine administration, de la transparence dans la prise de décision, et permettent aux citoyens et aux groupes concernés d'être consultés, voire d'y participer activement. L'honorable René Dussault, dans un article sur la relation de confiance entre l'État et les citoyens, écrivait en 2006 :

La consultation des milieux touchés par un programme ou par un projet et la connaissance de leur réaction face aux contraintes administratives imposées, en un mot, la juste appréciation des besoins réels et des perceptions des citoyens concernés sont devenues des éléments essentiels de succès et constituent autant d'efforts qui contribuent à concilier les nécessités administratives avec les droits des citoyens et à améliorer la qualité des relations entre ceux-ci et l'État.

On peut citer en exemple de nouvelles lois ou dispositions législatives, ainsi que de nombreux jugements (dont plusieurs de la Cour suprême du Canada), qui ont récemment posé les assises obligeant les responsables de la gouvernance des organisations publiques, mais aussi privées, à réaliser, voire à animer des prises de conscience, à assumer concrètement les responsabilités nouvelles assignées aux acteurs impliqués dans le développement. C'est notamment le cas des lois canadienne et québécoise sur le développement, et la loi anti-slapp, loi québécoise contre l'utilisation abusive des tribunaux pour empêcher les expres- sions d'opinion ou les publications. Ces lois favorisent le respect de la liberté d'expression et la participation des citoyens au débat public. De plus, les lois sur les sociétés par actions amènent les conseils d'administration à décider en fonction de l'intérêt à long terme de la société par actions, et leur permettent, voire même obligent, la prise en compte de l'intérêt de toutes les parties prenantes, dont la communautée ${ }^{27}$. La participation et l'engagement des citoyens sont représentés dans ces législations comme des principes de base, le développement durable impliquant en son principe même des changements de comportement, la sensibilisation de chacun, la participation de tous aux processus décisionnels; ces différents mécanismes de démocratie participative, devraient être un remède, soit dit en passant, à l'imprévoyance...

\section{Il existe au sein de l'administration publique de nombreux mécanismes favorisant l'exercice d'un contrôle. Certains mécanismes ne permettent d'agir qu'a posteriori, par exemple de faire une gestion de crise en cas de mauvaise administration, mais lorsque le mal est déjà fait, dirons-nous.}

Dans cette perspective, l'éthique du care (qui est fondamentalement une éthique de la bienveillance) ne sera plus l'apanage des seuls professionnels des soins physiques ou psychologiques. Elle appartiendra aussi aux gestionnaires qui doivent travailler à «maintenir, perpétuer et réparer notre "monde", de sorte que nous puissions y vivre aussi bien que possible ${ }^{28}$, reconstruisant ainsi les liens de confiance dans une société juste et équitable. Cette éthique prône un changement d'attitude vis-à-vis les êtres humains, les animaux, l'environnement. Fabienne Brugère explique que «le fait de "prendre soin" ou de pratiquer le care suppose que la relation n'est pas seulement une relation à, relation froide pourrait-on dire, mais une relation "chaude", ayant une profondeur temporelle, affective, et supposant de l'engagement personnel, de la compétence, de l'attention pour pouvoir exister comme telle ${ }^{29}$. Cette relation ne saurait exister si les rapports devenaient tout à fait distants, et seulement médiatisés par des logiques purement mercantiles ou instrumentales, celles qui servent à élaborer des processus ou des objets et à les échanger. 
Comme pratique généraliste, la tâche de l'administrateur est devenue souvent très complexe. Le terme de gouvernance (bien qu'il soit encore à l'occasion utilisé de façon réductrice) est plus adéquat pour représenter les perspectives et les orientations que les organisations doivent prendre en compte pour faire face aux défis à relever, à court, moyen et long terme.

Devant une telle tâche, un tel défi, le terme gestion ne suffit peut-être plus aujourd'hui à refléter tous les aspects de la pratique administrative dont l'ampleur se trouve mieux cernée par le terme de gouvernance. Comme pratique généraliste, la tâche de l'administrateur est devenue souvent très complexe. Le terme de gouvernance (bien qu'il soit encore à l'occasion utilisé de façon réductrice) est plus adéquat pour représenter les perspectives et les orientations que les organisations doivent prendre en compte pour faire face aux défis à relever, à court, moyen et long terme. Le concept de gouvernance traduit le fait que pèsent sur les stratégies décisionnelles des organisations une multitude de contraintes extérieures. Par exemple lorsqu'il s'impose d'animer et de refléter les relations de collaboration que les organisations doivent avoir avec l'ensemble des parties prenantes (clients, actionnaires, salariés, fournisseurs, ONG, gouvernements, communautés, etc.), dans une variété de contextes sociaux et institutionnels.

$\mathrm{Au}$ terme d'un processus international complexe, et sans précédent, de réflexion scientifique et éthique, ISO a réussi à publier, en 2010, la Norme ISO 26000 sur la responsabilité sociétale. Cette norme clarifie les principes directeurs de la vie collective et de la gouvernance des organisations, qu'elles soient privées ou publiques. La norme comporte un long développement sur les relations entre organisations et parties prenantes, qui rejoignent sur bien des points les aspects progressistes de nos lois en matière de participation, et le souci du rétablissement de la confiance. Selon ISO :

Il existe divers moyens permettant à une organisation d'établir sa crédibilité. L'un d'eux est le dialogue avec les parties pre- nantes qui est un moyen important d'améliorer la confiance dans le fait que les intérêts et les intentions de tous les participants sont bien compris. Ce dialogue permet d'établir la confiance et d'améliorer la crédibilité. Le dialogue avec les parties prenantes peut constituer la base de l'implication de ces dernières dans la vérification des déclarations d'une organisation concernant ses performances. L'organisation et les parties prenantes peuvent prendre des dispositions permettant à ces dernières de revoir périodiquement ou de surveiller d'une façon ou d'une autre certains aspects des performances d'une organisation $^{30}$.

\section{Au terme d'un processus international complexe, et sans précédent, de réflexion scientifique et éthique, ISO a réussi à publier, en 2010, la Norme ISO 26000 sur la responsabilité sociétale. Cette norme clarifie les principes directeurs de la vie collective et de la gouvernance des organisations, qu'elles soient privées ou publiques.}

C'est cette réalité, ce tout complexe, que négligent souvent les manuels de gestion traditionnels. En effet, les stratégies décisionnelles constituent désormais, selon Gilles Paquet, le produit d'une mission de stewardship ${ }^{31}$, garante d'une saine gouvernance. Il s'agit, selon nous, d'une mission dont la maîtrise doit devenir, dans un profil de carrière, une capacité progressive de l'administrateur ou du gestionnaire. Cette mission découle du fait que les pouvoirs, les façons de faire, les intérêts sont distribués entre de multiples centres décisionnels dans et hors de l'organisation contemporaine. L'administrateur ou le gestionnaire compétent doit savoir mobiliser ou prendre en compte ces facteurs multiples qui surdéterminent la production des services, des produits, des relations.

Les stratégies décisionnelles constituent désormais, selon Gilles Paquet, le produit d'une mission de stewardship, garante d'une saine gouvernance. Il s'agit, selon nous, d'une mission dont la maîtrise doit devenir, dans un profil de carrière, une capacité progressive de l'administrateur ou du gestionnaire. 


\section{L'INSTAURATION DE RAPPORTS DE CONFIANCE AVEC LES PARTIES PRENANTES : UNE QUESTION DE CONNAISSANCES ET DE TECHNIQUES BIEN MAÎTRISÉES}

\author{
L'instauration d'une confiance vigilante \\ entre toutes les parties prenantes, d'une \\ socialité marquée par l'intelligence \\ collective et le développement, requiert une \\ préparation adéquate des futurs \\ gestionnaires et professionnels.
}

L'instauration d'une confiance vigilante entre toutes les parties prenantes, d'une socialité marquée par l'intelligence collective et le développement, requiert une préparation adéquate des futurs gestionnaires et professionnels ${ }^{32}$. Ils auront un rôle fondamental à jouer afin d'écarter les modes de gestion qui affaiblissent les liens sociaux et réduisent les espaces d'autonomie.

Dans le schéma conceptuel présenté à la figure 1, il importe de voir arriver au centre du continuum des niveaux de confiance, toute une population de «s'éduquants », pourrions-nous dire, qui au départ pourrait très bien provenir des deux extrêmes, soit qu'ils présentent une confiance aveugle et imprudente aux événements, soit qu'ils affichent une méfiance systématique à leur égard. Pour changer efficacement de telles perceptions, il faudra former à l'esprit critique, à la compréhension des nombreux principes de la vie sociale (dont le rôle et l'influence des parties prenantes), aux instruments de collaboration (techniques d'animation de groupe, participation à des audiences publiques, etc.). Il faut aussi former aux processus éprouvés de délibération éthique. La mise à jour des programmes de formation permanente, de formation initiale, destinés aux gestionnaires, aux administrateurs et aux professionnels pourrait également s'inspirer de la norme ISO 26000.

Les silos disciplinaires empêchent encore très souvent de voir, d'utiliser l'expertise des autres. La compréhension des réalités sociologiques demeure très en retard, et de nombreux instruments d'analyse et outils d'intervention que les sciences sociales ont développés méritent qu'on s'y attarde ${ }^{33}$. Ces connaissances qui structurent une perspective multidimensionnelle sur la réalité des entreprises, des organisations, et qui permettent d'expliquer plus justement la réalité où elles interviennent, ne peuvent être réservées aux agents de développement et aux étudiants formés par les sciences humaines et sociales. Les gestionnaires ont tout intérêt à connaître ces expertises dans le contexte des profondes transformations qu'amènent, entre autres, la globalisation et le développement fulgurant des nouvelles technologies de communication.

Dans cette perspective, l'expérience française mérite d'être soulignée, car psychosociologues et sociologues y ont appris à intervenir sur les dimensions de la socialisation des individus et $\mathrm{du}$ fonctionnement des organisations. Lorsque des nœuds de tension, la perte de repères érodent la confiance, ou au pire, amènent une méfiance généralisée des acteurs d'une organisation et des parties prenantes, les interventions permettent d'appréhender globalement la complexité des rapports dans l'organisation, dans son environnement, de faire percevoir les contributions des différents acteurs, et plus globalement de mieux percevoir les liens entre toutes ces dimensions ${ }^{34}$. C'est alors que l'intervention éthique peut, à partir de la compréhension des logiques d'action et des références culturelles de l'organisation, faire sens, permettre de trouver une nouvelle articulation entre les fins et les moyens, bref, de faire émerger un consensus raisonnable sur une nouvelle base de coopération entre les parties, de provoquer la reconstruction de la confiance dans la durée $e^{35}$.

La mise à jour des programmes de formation permanente, de formation initiale, destinés aux gestionnaires, aux administrateurs et aux professionnels pourrait également s'inspirer de la norme ISO 26000. 


\section{CONCLUSION}

\section{La problématique de la confiance est cruciale, rappelait le sociologue Georg Simmel : «Sans la confiance des hommes les uns envers les autres, la société tout entière se disloquerait... ».}

Les organisations publiques ou privées peuvent difficilement toujours inspirer confiance, car elles ne sont pas morales en soi; elles sont d'ailleurs souvent animées par le souci du bienparaître. Par contre, les personnes physiques qui les dirigent, les gèrent, en assument la gouvernance, peuvent avoir un rôle prééminent dans la qualité des dialogues auxquels ils participent, dans le maintien du lien de confiance entre l'État, les organisations et les citoyens. La problématique de la confiance est cruciale, rappelait le sociologue Georg Simmel : «Sans la confiance des hommes les uns envers les autres, la société tout entière se disloquerait... ${ }^{36}$ Le problème contemporain est encore plus vaste; il s'agit de construire une société mondiale, ni plus ni moins, sur la base de sociétés particulières souvent menacées de disparition. Le temps des recettes et des modes en gestion est terminé.

\section{BIBLIOGRAPHIE ET NOTES}

${ }^{1}$ André Comte-Sponville, «La société en crise », Constructif, $\mathrm{n}^{\mathrm{O}} 11$, juin 2005.

${ }^{2}$ Juvenal, Satire VI, lignes 347-348.

${ }^{3}$ Joseph Stiglitz, Paul Krugman, Jeff Rubin, Jean Ziegler, Amartya Sen, parmi d'autres.

${ }^{4}$ En anglais, le terme confiance peut se traduire par deux mots : trust et confidence. "[...] trust paraît beaucoup plus fort que confidence car il inclut l'idée de faith, de foi, de croyance, alors que confidence [...] traduit une évaluation beaucoup plus objective des capacités à remplir une obligation et à établir une relation. » Jean-Michel Servet, «Le chapeau » dans Philippe Bernoux et Jean-Michel Servet (dir.), La construction sociale de la confiance, Paris, Montchrétien, 1997, p. 17, 26.

${ }^{5}$ Niklas Lühmann, La confiance, un mécanisme de réduction de la complexité sociale, Paris, Economica, Études sociologiques (coll.), 2006, p. 1.

${ }^{6}$ Gloria Origgi, Qu'est-ce que la confiance?, Paris, Vrin, 2008.

${ }^{7}$ On lira à ce sujet : Michael Kosfeld, Markus Heinrichs, Paul J. Zak, Urs Fischbacher, Ernst Fehr,
"Oxytocin increase trust in humans" Nature, vol. $435, n^{\circ} 2,2$ juin 2005, 673-676. Des critiques à ces études ne se feront pas attendre : Louis Quéré, "How could trust be restored to nature?", Intellectica, $\mathrm{n}^{\circ}$ 46-47, 2007, 105-122.

${ }^{8}$ On consultera, Canada, Code de valeurs et d'éthique de la fonction publique, Secrétariat du Conseil du trésor du Canada. On retrouve ce texte sur le site suivant : http://www.tbs-sct.gc.ca/pubs_pol/hrpubs/ TB_851/vec-cve01-fra.asp.

${ }^{9}$ Christian Thuderoz, Vincent Mangematin et Denis Harrison, La Confiance. Approches économiques et sociologiques, Gaëtan Morin, 1999; Michel Picard, Faites-moi confiance! : autopsie des crimes financiers, Montréal, Éditions Logiques, 2011.

${ }^{10}$ Alain Supiot, Homo Juridicus. Essai sur la fonction historique du droit, Paris, Seuil, 2005, pp. 157 à 160.

${ }^{11}$ Kenneth Arrow, "Gifts and exchanges", Philosophy and Public Affairs, vol. 1, 1972, p. 343-362, cité et traduit par Yann Algan, Pierre Cahuc, La société de défiance. Comment le modèle social français $s$ 'autodétruit, Paris, coll. CEPREMAP, Éditions Rue d'Ulm/Presses de l'École normale supérieure, 2007, pp. 88-9.

${ }^{12}$ Plusieurs ordres professionnels au Québec couvrent les activités en comptabilité, en gestion et en gestion des ressources humaines. D'autres professionnels interviennent très activement dans la gestion (ex. en gestion de projets) : les ingénieurs, les architectes, les notaires, les avocats, les évaluateurs agréés, les urbanistes, les agronomes et les géologues.

${ }^{13} \mathrm{CROP}$, Le Québec en mouvement. Tendances de fond pour 2012, Québec, septembre 2012. Ce sondage, cette fois, ne s'est pas restreint aux seuls professionnels assujettis au Code des professions. Il a touché d'autres activités qui ne sont pas prévues spécifiquement dans le Code, mais qui font partie des relations de service; le rôle des soldats, des pilotes d'avion, des enseignants, des éducatrices en garderie, des juges, des plombiers, des conseillers financiers, des journalistes, des mécaniciens, des chefs d'entreprise, des vendeurs automobile, des politiciens municipaux et nationaux.

${ }^{14} \mathrm{~L}$ 'enquête en ligne a été menée dans 25 pays auprès de 30000 répondants (soit environ 1000 personnes par pays) âgés de 25 à 64 ans et ayant un niveau d'information élevé des enjeux socioéconomiques. On consultera ce sondage à l'adresse suivante : http://trust.edelman.com/

${ }^{15}$ Olivier Cimelière, Baromètre Edelman Trust 2012 : Confiance, vous avez dit confiance?, Le Blog du Communicant 2.0.

${ }^{16}$ André Comte-Sponville rappelle la distinction entre la méfiance et la défiance : [...] le méfiant est 
incapable de confiance; le défiant ne donne sa confiance, comme dit Littré, "qu'après examen et réflexion". La méfiance, prise en ce sens, est une faiblesse; la défiance, ou, comme je préférerais dire, la vigilance, serait plutôt une vertu : c'est une occurrence de la prudence, dans les rapports à autrui, comme un refus légitime de se fier à ce qu'on ne connaît pas. André Comte-Sponville, «La société en crise », Constructif, $\mathrm{n}^{\circ} 11$, juin 2005.

${ }^{17}$ Robert K. Merton, Éléments de théorie et de méthode sociologique, Paris, Gérard Monfort, 1965, (Chapitre IV et son appendice); sur la prophétie autoréalisatrice en matière économique, on consultera Bernard Maris, Antimanuel d'économie, Paris, Éditions Bréal, 2003, pp. 306 et suiv.

${ }^{18}$ En 2008 , devant la ruée sur ses guichets, la banque Northern Rock n'a dû sa survie qu'à son rachat par la Banque d'Angleterre. La prophétie de faillite, une fausseté logique au départ, allait faire arriver ce qu'elle annonçait. La pensée magique n'est pas dénuée de sens, car elle produit du sens, et ce sens produit des effets réels. Pour enrayer ces effets sur les banques, Roosevelt avait compris l'importance d'instaurer l'assurance-dépôt, qui n'existe pourtant pas partout encore aujourd'hui !

${ }^{19}$ Louis Quéré dans Tarick Tazdaït, L'analyse économique de la confiance, Bruxelles, Éditions De Bœck Université, 2008, p. 14.

${ }^{20}$ Dans plusieurs codes de déontologie, on attend du professionnel qu'il soit toujours soucieux des conséquences de ses décisions (ou activités professionnelles), de ses travaux, de ses recherches, sur la société en général. S'ajoute, après le mot « conséquences », un qualificatif, « prévisibles », venant, en principe, limiter quelque peu la portée de l'obligation. Pour les disciplines de la gestion, on consultera, au Québec, les codes de déontologie des administrateurs agréés, des conseillers en ressources humaines et en relations industrielles, des évaluateurs agréés.

${ }^{21}$ Le concept de développement, d'abord foyer de réflexion dans les sciences de la vie, a essaimé et s'est trouvé à alimenter les sciences sociales, la sociologie et la science économique en particulier. Dans Le scandale du développement, l'économiste français Jacques Austruy proposait une définition du concept que nous pouvons actualiser en y intégrant des considérations environnementales ou d'équité, des dimensions propres à une gouvernance fiduciaire responsable. Ainsi, dans nos propres termes, le développement se conçoit comme l'ensemble des transformations institutionnelles, psychosociales, mentales qui ouvrent l'accès à une croissance pérenne, à une diminution des inégalités, au respect des cultures particulières, en symbiose avec l'environnement. On consultera Jacques Austruy, Le scandale du développement : commentaires par $G$. Leduc et L.-J. Lebret. Bibliographie analytique et critique par Guy Caire. Nouv. éd. corr. et mise à jour, Paris, M. Rivière, 1972.

${ }^{22}$ Amartya Sen, L'idée de Justice, Paris, Flammarion, 2010; Martha Nussbaum, Capabilités. Comment créer les conditions d'un monde plus juste? Paris, Climats, 2012.

${ }^{23}$ Yann Algan, Pierre Cahuc, André Zylberberg, La fabrique de la défiance, Paris, Albin Michel, 2012; Anne Gratacap, Alice Le Flanchec, La confiance en gestion : un regard pluridisciplinaire, Bruxelles, De Bœck, 2011.

${ }^{24}$ Gilles Paquet, Gouvernance et éthique, Résumé pour Info Adm. A. de l'allocution d'ouverture prononcée à l'occasion du Congrès annuel de 2012 de l'Ordre des administrateurs agréés du Québec, Centre Mont-Royal de Montréal, le 24 janvier 2012.

${ }^{25}$ André Comte-Sponville, «La société en crise », Constructif, $\mathrm{n}^{\circ} 11$, juin 2005.

${ }^{26}$ René Dussault, «L'État et les citoyens : une relation de confiance à renforcer ", Revue générale de droit, vol. 36, 2006, 579-589.

${ }^{27}$ Le gouvernement du Québec a adopté une loi sur l'intégrité en matière de contrats publics (2012, chapitre 25) qui prévoit que l'Autorité des marchés financiers (AMF) pourra refuser d'accorder une autorisation à une entreprise souhaitant transiger avec l'État «si elle considère que la confiance du public est affectée ».

${ }^{28}$ Joan Tronto, Un monde vulnérable. Pour une politique du care, Paris, Éditions La Découverte, 2009, p. 143.

${ }^{29}$ Fabienne Brugère, «Jusqu'où ira le care ? », La Vie des idées, 4 octobre 2010. ISSN : 2105-3030. URL : http://www.laviedesidees.fr/Jusqu-ou-ira-lecare.html

${ }^{30}$ International Standard Organisation (ISO), Guidance of Social Responsability /Lignes directrices relatives à la responsabilité sociétale, ISO 26000, 2010, $1^{\text {ère }}$ édition, p. 95.

${ }^{31}$ Voir à ce sujet, Gilles Paquet, Gouvernance et éthique, Résumé pour Info Adm. A. de l'allocution d'ouverture prononcée à l'occasion du Congrès annuel de 2012 de l'Ordre des administrateurs agréés du Québec, Centre Mont-Royal de Montréal, le 24 janvier 2012. On consultera aussi Gilles Paquet, La gouvernance collaborative, Montréal, Liber, 2011.

${ }^{32}$ Yann Algan, Pierre Cahuc, André Zylberberg, La fabrique de la défiance, Paris, Albin Michel, 2012.

${ }^{33}$ Pour une description des différents outils et techniques d'animation participative, on consultera : Cheikh Sow, Mehdi Hazgui, «Catalogue des outils et techniques d'animation participative », mars 2011.

${ }^{34}$ Marie-Hélène Cabé, Robert-Tanguy Philippe, «L'intervention sociologique : (re)donner du sens à 
des pratiques gestionnaires », Sociologies pratiques, vol. $1 \mathrm{n}^{\circ}$ 16, 2008, 39-54; Hélène Delahaye, «Sociologue en entreprise : réflexions sur la complémentarité des savoirs ", Sociologies pratiques, vol.1, $\mathrm{n}^{\circ}$ 16, 2008, 55-62; F. Osty, R. Sainsaulieu, Marc Uhalde, Les mondes sociaux de l'entreprise, Paris, La Découverte, 2007; Renaud Sainsaulieu, «Préface», dans L'intervention sociologique en entreprise. De la crise à la régulation sociale, Marc Uhalde (dir.), Paris, Desclée de Brouwer, 2001, p. 11; Marc Uhalde, «Quand gestion rime avec confusion», Sciences humaines, $\mathrm{n}^{\circ} 179$, février 2007.

${ }^{35} \mathrm{Il}$ y a une abondante littérature à ce sujet. Sur le rôle de la délibération éthique sur l'amélioration de la confiance dans les organisations publiques et privées, on consultera, Luc Bégin, «Un conflit des identités? La régulation par l'éthique en milieu de travail», dans Qu'est-ce qu'une société d'individus?, S. Charles, P. H. Tavoillot (dir.), Montréal, Liber, 2007, p. 137-154; Yves Boisvert, «Crise de confiance et crise de légitimité : de l'éthique gouvernementale à l'éthique publique », Éthique publique, vol. 4, n ${ }^{\circ}$ 1, 2007.

${ }^{36}$ Georg Simmel, Philosophie de l'argent, Paris, PUF, 1987, p. 197. 